
capture of SARS-CoV-2 RNA from wastewater.

3 Oscar N. Whitney ${ }^{1}$, Lauren C. Kennedy ${ }^{2}$, Vinson Fan ${ }^{1}$, Adrian Hinkle ${ }^{2}$, Rose Kantor ${ }^{2}$, Hannah

4 Greenwald $^{2}$, Alexander Crits-Christoph ${ }^{3,4}$, Basem Al-Shayeb $^{3,4}$, Mira Chaplin ${ }^{2}$, Anna C.

5 Maurer ${ }^{1}$, Robert Tjian ${ }^{1,5}$, Kara L. Nelson ${ }^{2,4+}$

6 +Corresponding author: karanelson@berkeley.edu

$7 \quad{ }^{1}$ Department of Molecular and Cell Biology, University of California, Berkeley, CA, USA

$8{ }^{2}$ Department of Civil and Environmental Engineering, University of California, Berkeley, CA, 9 USA

$10{ }^{3}$ Department of Plant and Microbial Biology, University of California, Berkeley, CA, USA

$11{ }^{4}$ Innovative Genomics Institute, Berkeley, CA, 94704, USA

$12{ }^{5}$ The Howard Hughes Medical Institute, University of California Berkeley, Berkeley, California 1394720, USA

14 ABSTRACT:

Wastewater-based epidemiology is an emerging tool to monitor COVID-19 infection

16 levels by measuring the concentration of severe acute respiratory syndrome coronavirus 2

17 (SARS-CoV-2) RNA in wastewater. There remains a need to improve wastewater RNA

18 extraction methods' sensitivity, speed, and reduce reliance on often expensive commercial

19 reagents to make wastewater-based epidemiology more accessible. We present a kit-free

20 wastewater RNA extraction method, titled "Sewage, Salt, Silica and SARS-CoV-2” (4S), that

21 employs the abundant and affordable reagents sodium chloride $(\mathrm{NaCl})$, ethanol and silica $\mathrm{RNA}$

22 capture matrices to recover 6-fold more SARS-CoV-2 RNA from wastewater than an existing 
23 ultrafiltration-based method. The 4S method concurrently recovered pepper mild mottle virus

24 (PMMoV) and human 18S ribosomal subunit rRNA, both suitable as fecal concentration

25 controls. The SARS-CoV-2 RNA concentrations measured in three sewersheds corresponded to

26 the relative prevalence of COVID-19 infection determined via clinical testing. Lastly, controlled

27 experiments indicate that the $4 \mathrm{~S}$ method prevented RNA degradation during storage of

28 wastewater samples, was compatible with heat pasteurization, and could be performed in

29 approximately 3 hours. Overall, the $4 \mathrm{~S}$ method is promising for effective, economical, and

30 accessible wastewater-based epidemiology for SARS-CoV-2, providing another tool to fight the

31 global pandemic.

32 KEYWORDS: Wastewater-based epidemiology, SARS-CoV-2, COVID-19, Wastewater RNA

33 extraction, direct extraction, ultrafiltration, heat pasteurization

34 SYNOPSIS: The 4S method for measuring SARS-CoV-2 in wastewater is promising for

35 effective, economical, and accessible wastewater-based epidemiology.

\section{ABSTRACT ART:}

$\mathrm{NaCl}$-mediated wastewater lysis
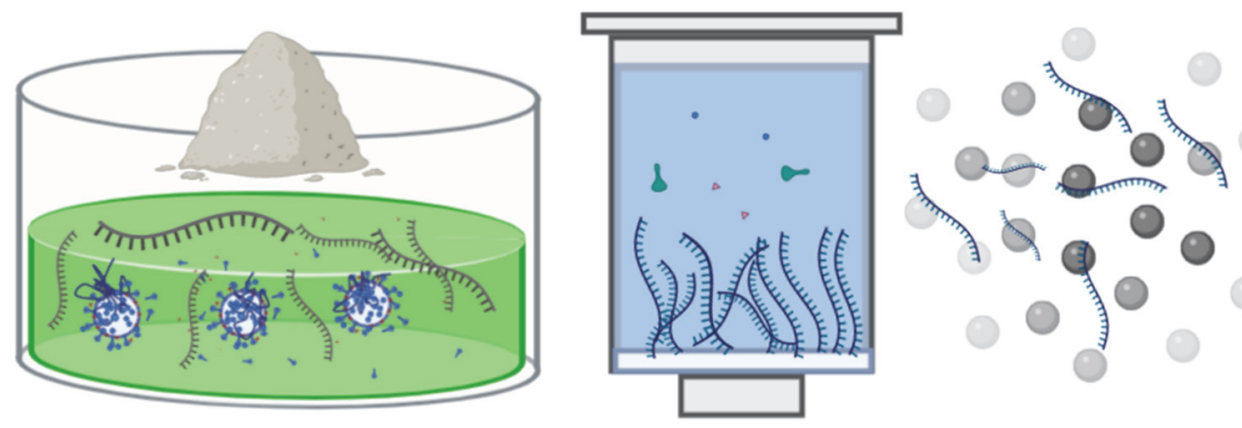

Silica column or Silica particulate
SARS-CoV-2 signal assessment

(1)

(1) 
Wastewater-based epidemiology (WBE) enables the indirect assessment of viral infection

39 prevalence in populations. ${ }^{1-3}$ The quantity of viral nucleic acids shed into wastewater by infected

40 individuals, whether symptomatic or not, serves as a proxy for the relative prevalence of

41 infection. ${ }^{1}$ WBE can provide population-level infection information for up to many thousands of

42 individuals in a community to complement individual-level testing and aid public health decision

43 making. 4

WBE is now being applied to monitor and even predict population-level coronavirus disease 2019 (COVID-19) outbreaks. ${ }^{1,5}$ Local COVID-19 prevalence is difficult to assess due to

46 insufficient individual testing capacity, rendering effective response more challenging. ${ }^{6}$

47 Wastewater can provide insights into COVID-19 prevalence, as COVID-19 patients shed SARS-

48 CoV-2 RNA in their stool and thus into wastewater. ${ }^{7,8}$ Emerging studies report wastewater

49 SARS-CoV-2 concentrations that correspond to reported clinical prevalence of COVID-19, with

50 potential for early detection of COVID-19 outbreaks and identification of newly emerging

51 SARS-CoV-2 variants. $^{9-12}$ To extract and quantify the concentration of SARS-CoV-2 RNA shed

52 into wastewater, researchers are using size- and charge-based concentration methods that

53 concentrate intact SARS-CoV-2 virus prior to RNA extraction. ${ }^{13-15}$ These methods employ a

54 primary concentration step via sieving by particle size, enmeshment of viral particles in

55 precipitates that can be separated by mass, or adsorption via electrostatic interactions, prior to

56 RNA extraction. ${ }^{15}$ These methods are relatively time-consuming and inaccessible as they are

57 dependent on reliable supply of commercial reagents, a paucity of which has already hampered

58 clinical SARS-CoV-2 testing efforts. ${ }^{13-16}$ Further, the use of primary concentration assumes the

59 recovery of intact virus, and is therefore not geared towards co-capturing RNA from SARS-

60 CoV-2 viruses that have already lysed or capture of non-viral RNAs suitable as fecal 
61 concentration controls. ${ }^{14}$ Lastly, current CDC safety guidelines recommend BSL-3 precautions

62 when employing environmental sampling procedures that concentrate viruses presumed to be

63 intact. ${ }^{17}$ To mitigate concerns of concentrating potentially infectious virus, heat-based

64 wastewater sample pasteurization and subsequent extraction could allow for easier and safer

65 wastewater processing after collection.

We aimed to develop an economical, kit-free method for the direct capture (extraction) of

67 SARS-CoV-2 RNA from wastewater. The method employs lysis of biological particles via

68 sodium chloride $(\mathrm{NaCl})$, heat-based pasteurization, coarse filtration, ethanol precipitation, and

69 RNA capture via silica-based columns (4S-column) or silicon dioxide slurry (4S-Milk-of-Silica).

70 This approach allows recovery of wastewater RNA without mass, size, or charge bias and the co-

71 capture of RNA from lysed SARS-CoV-2 virus alongside RNA from other biological particles in

72 wastewater that are suitable as fecal concentration controls, such as pepper mild mottle virus

73 (present in dietary peppers and shed in feces) and human $18 \mathrm{~S}$ ribosomal RNA. ${ }^{18}$ The $4 \mathrm{~S}$ method

74 stabilizes the nucleic acids in wastewater via the addition of sodium chloride $(\mathrm{NaCl})$ and

75 ethylenediaminetetraacetic acid (EDTA), and is compatible with heat pasteurization, which

76 makes wastewater samples safer to process. The 4S method's omission of a primary

77 concentration step and kit-free extraction enables lower reliance on commercial reagents and

78 speeds up RNA purification to enable same-day measurement of SARS-CoV-2 wastewater

79 abundance. 


\section{MATERIALS AND METHODS:}

\section{Sample collection}

85 For this study, we obtained composite 24-hour wastewater influent samples from East Bay

86 Municipal Utility District's wastewater treatment plant. These samples represent three discrete

87 sampling areas: North and west Berkeley, El Cerrito, Kensington and Albany (sub-sewershed

88 "N"), Oakland/Piedmont (sub-sewershed "S"), and Berkeley/Oakland Hills (sub-sewershed "A")

89 (interceptor coverage detailed in Fig. S2A). Samples were kept at $4^{\circ} \mathrm{C}$ on ice during transport

90 and processed within 24 hours or kept at $-80^{\circ} \mathrm{C}$ and processed within two weeks.

\section{Wastewater RNA extraction}

92 Wastewater RNA extraction via the 4S-column and 4S-Milk-of-Silica methods is detailed in

93 depth at https://www.protocols.io/view/v-4-direct-wastewater-rna-capture-and-purification-

94 bpdfmi3n and $\underline{\mathrm{dx} . \text { doi.org/10.17504/protocols.io.biwfkfbn }}{ }^{28,29}$. In brief, for 4S RNA extraction

95 using a silica column, samples were lysed via the addition of Sodium Chloride $(\mathrm{NaCl})$ to a final

96 concentration of $4 \mathrm{M}$ and EDTA to a final concentration of $1 \mathrm{mM}$ and buffered via the addition

97 of $10 \mathrm{mM} \mathrm{pH} 7.2$ tris(hydroxymethyl)aminomethane. Samples were heat inactivated in a water

98 bath (unless indicated otherwise) at $70^{\circ} \mathrm{C}$ for 45 minutes, filtered using a $5-\mu \mathrm{M}$ DuraPore PVDF

99 filter membrane (Millipore Sigma) and syringe filter. Ethanol was added to sample filtrate to a

100 final concentration of 35\%. Samples were passed through Zymo-IIIP silica columns (Zymo

101 Research) using a vacuum manifold. For all experiments other than the wash buffer tests (Figure

102 4, Supplemental Figure S4), samples were washed with $25 \mathrm{~mL}$ of high $\mathrm{NaCl}(1.5 \mathrm{M})$ and ethanol

$103(20 \%)$ containing wash buffer \#1 (4S-WB1), and $50 \mathrm{~mL}$ of low $\mathrm{NaCl}(100 \mathrm{mM})$ and ethanol

104 (80\%) containing wash buffer \#2 (4S-WB2). Washed RNA was eluted from silica columns using 
$105200 \mu \mathrm{L}$ of ZymoPURE elution buffer (Zymo Research) or $\mathrm{pH} 8$ Tris-EDTA buffer pre-heated to $10650^{\circ} \mathrm{C}$.

107 For 4S-Milk-of-Silica extraction, samples were lysed, heat inactivated and filtered as in the 4S-

108 column extraction. Next, a $1 \mathrm{~g} / \mathrm{mL}$ silicon dioxide slurry in water was added to the filtered lysate

109 and incubated at room temperature for 10 minutes. The lysate and silica slurry were centrifuged

110 at $4000 \mathrm{x}$ g for 5 minutes, pelleting wastewater RNA bound to silica particulate. The lysate

111 supernatant was decanted, and the silica pellet was washed with $40 \mathrm{~mL} 4 \mathrm{~S}-\mathrm{WB} 1$ and $40 \mathrm{~mL}$ of

112 4S-WB2 via centrifugation and wash buffer decanting. The washed silica pellet was resuspended

113 in $20 \mathrm{~mL}$ of pure water pre-heated to $37^{\circ} \mathrm{C}$ to elute bound RNA. Next, the silicon dioxide

114 particulate was pelleted via centrifugation and the eluted RNA was separated and concentrated

115 via isopropanol precipitation, as previously described. ${ }^{30,29} 4$ S-column and 4S-Milk-of-Silica

116 reagent costs are listed in Supplementary Table 6.

117 For sample RNA concentration via ultrafiltration, Amicon 100-kDa ultrafilters (Millipore Sigma)

118 were pretreated to block virus adsorption using $2 \mathrm{~mL}$ bovine serum albumen 1\% (w/v) in $1 \mathrm{x}$ PBS

119 and then washed with PBS. Wastewater samples were divided into $40 \mathrm{~mL}$ aliquots and solids

120 were removed via slow centrifugation with a swinging bucket rotor at $4700 \mathrm{x}$ g for $30 \mathrm{~min}$.

121 Supernatant was decanted and passed through a $0.2 \mu \mathrm{m}$ flat membrane filter (Steriflip, EMD

122 Millipore). Filtrate was loaded onto the ultrafilter in increments of up to $15 \mathrm{~mL}$ and ultrafilters

123 were spun for 10 minutes at $4700 \mathrm{xg}$ for each increment. Flow-through was discarded and

124 samples were concentrated until they were reduced to a final volume of $\sim 250 \mu 1$. RNA was

125 extracted from the ultrafiltration concentrate using an AllPrep DNA/RNA Mini kit (QIAGEN)

126 following manufacturer instructions. 


\section{RNA detection and quantification via RT-qPCR}

128 This study employed four primer/probe sets: the SARS-CoV-2 N1 assay, Pepper mottle mild

129 virus (PMMoV) coat protein gene assay, bovine coronavirus transmembrane protein gene assay

130 and a newly developed human 18S ribosomal rRNA assay. (Supporting information, Table S3.)

131 RT-qPCR reaction conditions are detailed in Table S1, assay thermocycling conditions are

132 detailed in Table S2, and primer sequence information is in Table S3. RT-qPCR assay

133 performance is detailed in Table S4 (Validation) and Table S5 (Limit of detection). RT-qPCR

134 minimum information for publication of quantitative real-time PCR experiments (MIQE)

135 documentation is detailed in Table S7. RT-qPCR analysis is detailed in the supporting

136 information.

\section{RESULTS AND DISCUSSION:}

Many current methods of wastewater viral RNA extraction assume that most viral

139 particles within wastewater are intact and that the concentration of these intact viruses prior to

140 extraction is necessary to achieve sensitive detection of SARS-CoV-2 in wastewater. Given this

141 assumption, these methods typically employ precipitation-, charge-, or size-based viral

142 concentration and subsequent RNA extraction of unpasteurized wastewater to preserve viruses in

143 an intact state. ${ }^{14,15}$ Despite concentration, some methods were shown to recover as little as $0-1 \%$

144 of SARS-CoV-1 from wastewater during the SARS-CoV-1 epidemic. ${ }^{19}$ We hypothesized that

145 direct extraction could avoid loss of virus during the primary concentration step, and we

146 therefore designed the 4S (Sewage, Salt, Silica and SARS-CoV-2) method to lyse viruses and

147 microorganisms present in wastewater using sodium chloride and subsequently capture the free

148 RNA using a silica RNA binding matrix. 
medRxiv preprint doi: https://doi.org/10.1101/2020.12.01.20242131; this version posted December 3, 2020. The copyright holder for this preprint

(which was not certified by peer review) is the author/funder, who has granted medRxiv a license to display the preprint in perpetuity.

It is made available under a CC-BY-NC-ND 4.0 International license .

To benchmark the performance of the $4 \mathrm{~S}$ method, we analyzed a 24-hour composite

150 wastewater sample treated with and without heat pasteurization and compared the recovery of

151 endogenous SARS-CoV-2 to that of an ultrafiltration-based method. In addition, we compared

152 the recovery of indigenous pepper mild mottle virus (PMMoV) RNA, which may be useful to

153 control for variable fecal concentrations in wastewater, and a spiked-in bovine coronavirus

154 vaccine (bCoV), used as an RNA extraction process control (Figure 1). We observed that the

155 4S-column method recovered 6-fold more SARS-CoV-2 RNA than ultrafiltration (Figure 1). 
medRxiv preprint doi: https://doi.org/10.1101/2020.12.01.20242131; this version posted December 3, 2020. The copyright holder for this preprint (which was not certified by peer review) is the author/funder, who has granted medRxiv a license to display the preprint in perpetuity.

It is made available under a CC-BY-NC-ND 4.0 International license.

Figure 1. Comparison of SARS-CoV-2, $\mathrm{PMMoV} \& \mathrm{bCoV}$ spike-in assay signal in gene copies per liter between the 4 S-column method with and without heat inactivation, and ultrafiltration. " $\mathrm{n}$ " represents the number of wastewater RNA extraction replicates per condition. Bars are plotted at the geometric mean of biological triplicates and error bars represent the variation associated with biological triplicates as quantified by the geometric standard deviation of the biological triplicates.

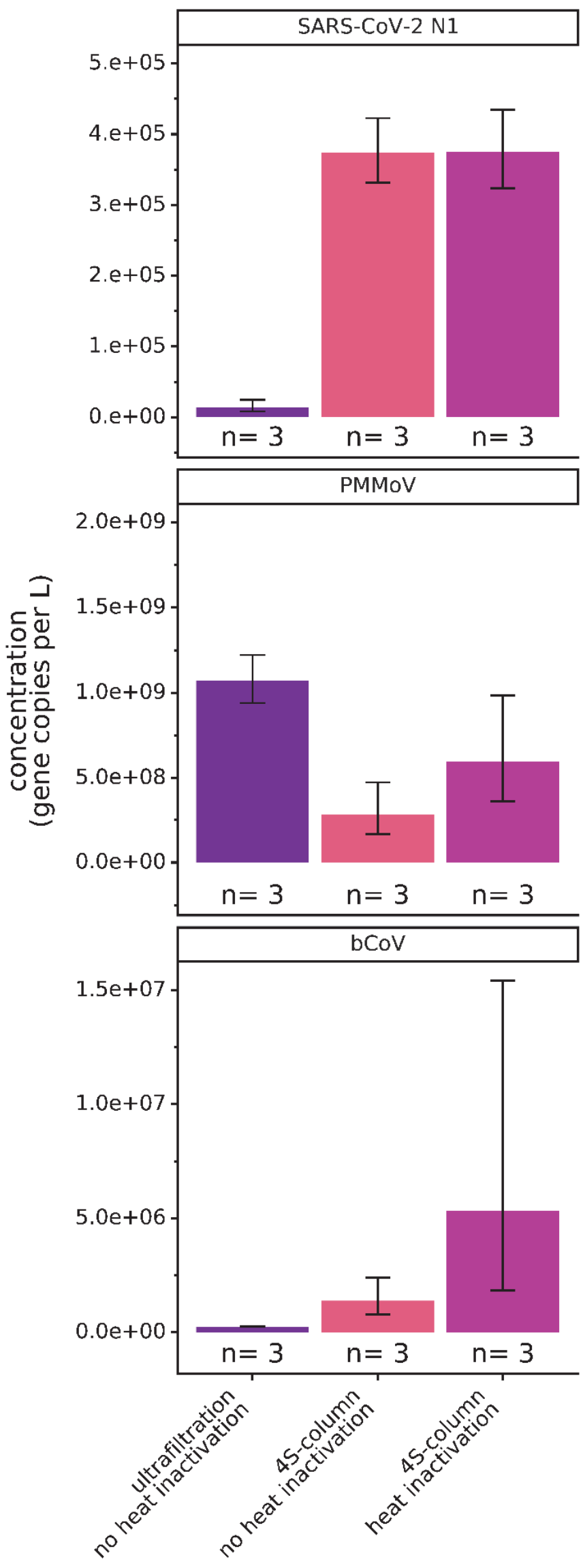


158 pasteurization, suggesting that further SARS-CoV-2 virus lysis did not occur. This result may

159 imply that a large fraction of SARS-CoV-2 RNA was not bound to virus particles; this unbound

160 RNA was captured by the 4S method but was not efficiently concentrated by ultrafiltration.

162 than ultrafiltration, and 28 -fold more bCoV with heat pasteurization. In this case, heat

163 pasteurization may promote additional lysis of encapsidated bCoV, releasing its RNA for

164 subsequent capture. Recovery of PMMoV by 4S was also higher with heat pasteurization (2-fold

165 increase in recovery), but ultrafiltration was more effective in enriching PMMoV (1.6-fold

166 higher than using the 4S-column method with heat pasteurization). Here, ultrafiltration may be

167 effective in concentrating intact virus that is able to persist in wastewater, which is consistent

168 with previous reports on PMMoV. ${ }^{18,20}$ Collectively, these results suggest that a significant

169 fraction of SARS-CoV-2 RNA in the analyzed wastewater was not bound to viral particles but

170 was present as free or ribonucleoprotein-bound RNA. This possibility is consistent with reports

171 indicating reduced viability of SARS-CoV-2 and related coronaviruses spiked into

172 wastewater. $^{13,21}$

173 Given that the 4 S method is designed to lyse and extract wastewater RNAs without

174 requiring the enrichment of viral particles, we also investigated whether the 4S method could

175 recover human RNAs present in wastewater. Using the 4S method, we were able to recover and

176 detect human ribosomal subunit RNA (18S rRNA) in wastewater influent (Supplementary Figure

177 S1A). 18S rRNA recovery was enhanced 2.5-fold by heat pasteurization, suggesting the lysis of

178 human cells or $18 \mathrm{~S}$ rRNA bound to ribonucleoprotein complexes present in wastewater

179 (Supplementary Figure S1A). Therefore, the 4S method enabled the recovery and detection of 
180 human RNA, another potential indicator of wastewater fecal concentration, which could allow

181 direct normalization of SARS-CoV-2 RNA quantity to human RNA content of wastewater. As

182 heat pasteurization did not affect $4 \mathrm{~S}$ recovery of SARS-CoV-2 and improved the recovery of

183 PMMoV, bCoV and 18S rRNA, we recommend integrating this pathogen inactivation step to

184 increase the safety of processing wastewater samples.

We sought to adapt the 4S strategy to employ silica powder for RNA capture rather than silica columns to circumvent reliance on commercially manufactured silica columns. In this

187 approach, we added a slurry of silicon dioxide particles to lysed wastewater and used

188 centrifugation to separate particle-bound RNA from the wastewater matrix, an approach we

189 named "4S-Milk-of-Silica". We observed that the 4S-Milk-of-Silica method recovered

190 equivalent SARS-CoV-2 and PMMoV signal to the 4S method using a silica column

191 (Supplementary Figure S1B). Thus, the 4S-Milk-of-Silica method presents an even more cost-

192 effective ( $\sim 8$ per sample, vs. $\sim \$ 13$ per sample, using the 4S-column extraction method,

193 Supplementary Table 6) and accessible method to extract wastewater RNA without reliance on

194 commercially manufactured silica columns and a vacuum manifold. However, the "Milk of

195 Silica" version of the 4S protocol requires an isopropanol precipitation RNA concentration step,

196 lengthening the protocol time. Therefore, we recommend using the 4S-column method to enable

197 faster sample processing, while "4S-Milk-of-Silica” presents an alternate protocol for use in 198 resource-limited settings.

200 prevalence, so we assessed whether the 4S-column extraction method could detect differential

201 SARS-CoV-2 RNA levels in wastewaters derived from different subsections of a collection

202 system. We surveyed three wastewater influent interceptors serving North and West Berkeley 
204 shown in Supplementary Figure S2A). These interceptors served areas exhibiting differential

205 incidence of clinically confirmed COVID-19 cases, ranging from three (A interceptor) to 68 (S

206 interceptor) reported cases per day within the week of our sampling (Figure S2A). To compare

207 clinical COVID-19 case data and wastewater SARS-CoV-2 concentration, we normalized the

208 case data by population, and we normalized the SARS-CoV-2 quantity by PMMoV abundance,

209 to control for fecal concentration in the wastewater. Raw SARS-CoV2 and PMMoV abundance

210 is available in Supplementary Figure 2B. As expected, the normalized wastewater SARS-CoV-2

211 signals trended with the per capita clinical cases per day in the three sub-sewersheds (Figure 2,

212 B). The normalized SARS-CoV-2 RNA concentration was highest in wastewater representing

213 the S interceptor area, where the highest daily per capita new cases also occurred. Normalized

214 SARS-CoV-2 RNA concentrations in wastewaters representing the N interceptor area were only

215 2.3-fold lower than those of S interceptor wastewaters, despite 11.6-fold fewer per capita daily

216 cases being reported in the A interceptor area during the week of our sample collection. One

217 possible reason for this difference could be the presence of undiagnosed infections in the $\mathrm{N}$

218 interceptor service area, in which case wastewater SARS-CoV-2 RNA concentrations may

219 provide a more accurate view of the relative COVID-19 infection prevalence in the week prior to

220 sampling. Alternatively, the variability associated with wastewater measurements may be too

221 large to detect differences of this magnitude. ${ }^{9,13}$ Ongoing research seeks to better quantify the

222 measurement variability in wastewater samples over temporal and spatial scales. We emphasize

223 that SARS-CoV-2 RNA levels were quantifiable in the A sub-sewershed despite only 18 cases

224 being reported in an estimated population of 90,000 during the weeklong period of our sampling. 
medRxiv preprint doi: https://doi.org/10.1101/2020.12.01.20242131; this version posted December 3, 2020. The copyright holder for this preprint (which was not certified by peer review) is the author/funder, who has granted medRxiv a license to display the preprint in perpetuity.

It is made available under a CC-BY-NC-ND 4.0 International license .

225 This result implies that the 4S method is highly sensitive and can be used to monitor areas with

226 low COVID-19 prevalence.

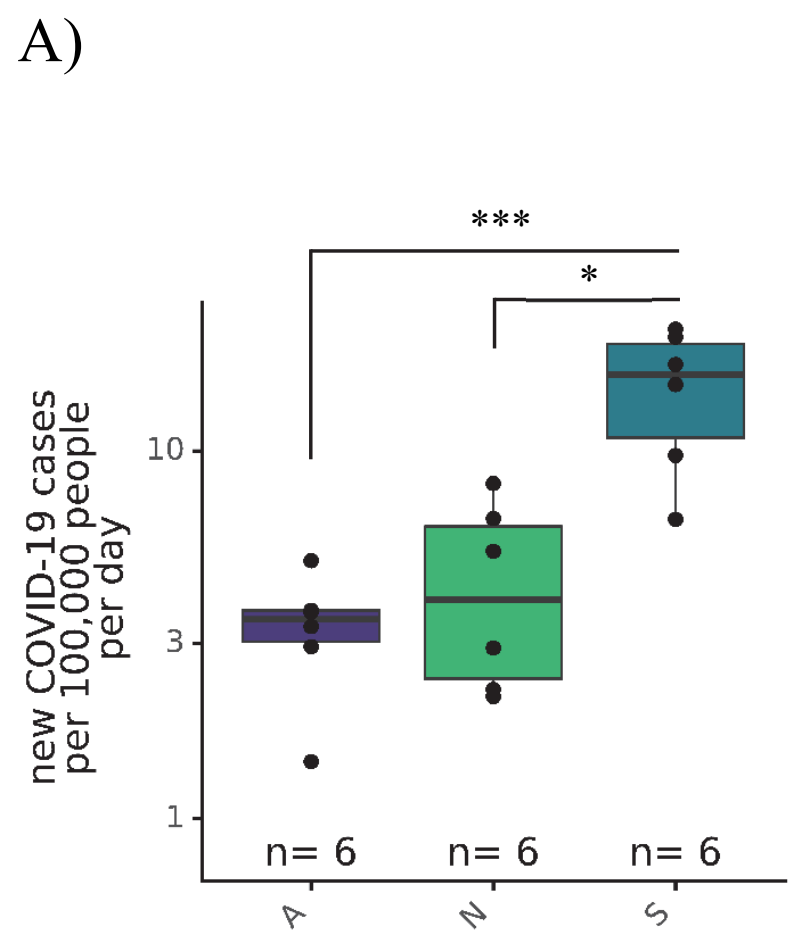

B)

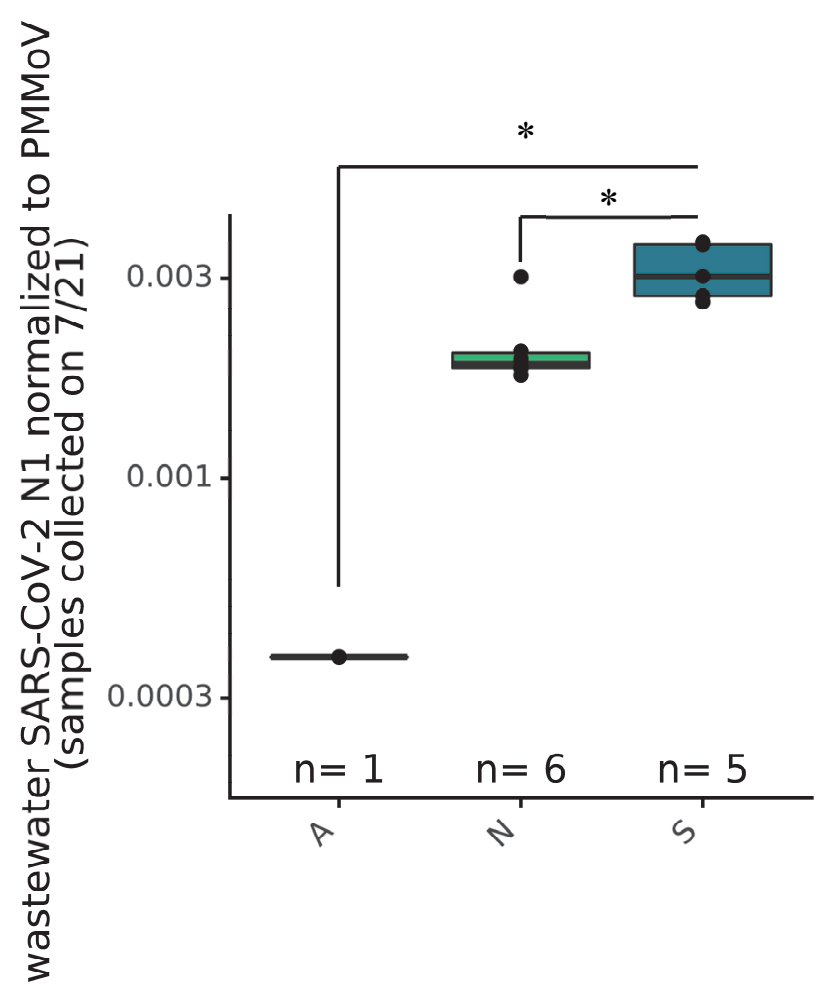

Figure 2. A) New COVID-19 cases per day per 100,000 population in three areas served by the distinct A, $\mathrm{N}$ and $\mathrm{S}$ wastewater interceptors over 6 days from 7/15 to 7/21. B) Comparison of SARS-CoV-2 N1 assay represented as SARS-CoV-2 gene copies per liter normalized to PMMoV gene copies per liter between interceptors serving the A, N and S East Bay areas. Kruskal-Wallis test followed by Dunn's test was performed to determine significance, where $*=p<0.05$ and $* * *=p<0.001$.

Wastewater contains many contaminants with the potential to degrade nucleic acids, and

228 it has been previously observed that SARS-CoV-2 RNA in wastewater is degraded during

229 storage. ${ }^{22-24}$ Viral detection relying on wastewater RNA extraction methods that concentrate

230 intact viruses may be strongly affected by variable amounts of virus and viral RNA degradation

231 in wastewater. Therefore, we sought to assess whether EDTA and sodium chloride, added to

232 wastewater in the $4 \mathrm{~S}$ method to promote lysis, could dually act to preserve RNA in wastewater. 
233 Upon receipt of each wastewater sample, we added $\mathrm{NaCl}$ to a final concentration of $4 \mathrm{M}$, added

234 EDTA to a final concentration of $1 \mathrm{mM}$, and stored the samples either at $4^{\circ} \mathrm{C}$ for a month, or

235 three days at room temperature $\left(20^{\circ} \mathrm{C}\right)$. We observed that salt and EDTA addition prior to

236 storage improved SARS-CoV-2 N1 assay signal after storage at both $4{ }^{\circ} \mathrm{C}$ for one month (2.6-

237 fold higher signal when stored with salt and EDTA) or at $20^{\circ} \mathrm{C}$ for three days (22-fold higher

238 signal when stored with salt and EDTA) (Figure 3). Interestingly, the PMMoV assay signal

239 remained similar throughout storage with or without salt, implying that PMMoV remains

240 resistant to RNAses in the wastewater matrix. This observation corroborates previous reports

241 indicating the persistence of PMMoV in wastewater. ${ }^{18,20}$ As with SARS-CoV-2 N1 signal, we

242 observed that salt and EDTA addition preserved human $18 \mathrm{~S}$ rRNA signal at $4^{\circ} \mathrm{C}$ for one month

243 (126-fold higher) or at $20^{\circ} \mathrm{C}$ for three days (56-fold higher) (Figure 3). These results again

244 support the conclusion that much of the SARS-CoV-2 in wastewater is not bound by intact

245 capsid, rendering it more susceptible to degradation, unlike PMMoV which may remain

246 encapsidated to protect it from degradation. Overall, the lysis salts added to wastewater as part of

247 the normal 4S method workflow conveniently preserved wastewater RNAs and may mitigate

248 degradation-mediated variation in SARS-CoV-2 and fecal concentration controls caused by RNA

249 degradation during shipping and storage.

Given the impact of RNA degradation on SARS-CoV-2 N1 assay signal, we investigated

251 whether bulk RNA yield, representing intact wastewater RNA, could be employed as a

252 normalization measure for SARS-CoV-2 detection. Surprisingly, bulk RNA yield per mL of

253 wastewater input correlated poorly with SARS-CoV-2 and PMMoV detection (Supplementary

254 Figure 3A). These results imply that most bulk wastewater RNA may be contributed by

255 wastewater microorganisms unrelated to human fecal content or viral content, thus weakly 
256 correlating to SARS-CoV-2 N1 assay signal. We also observed that extracting nucleic acids from

257 increasing volumes of wastewater (up to $400 \mathrm{~mL}$ ) did not strongly increase total RNA yield per

258 extraction past $100 \mathrm{~mL}$ of wastewater sample input, implying potential saturation of the RNA

259 capture matrix (Supplementary Figure S3B). From these experiments, we conclude that the RT-

260 qPCR detection of human fecal concentration indicators such as PMMoV and human 18S rRNA,

261 the latter of which is preserved during storage similarly to SARS-CoV-2, are better estimators of

262 wastewater fecal concentration than bulk RNA quantity measurements. Lastly, we observed that

263 the $4 \mathrm{~S}$ method enriched up to $8 \mu \mathrm{g}$ of DNA per $100 \mathrm{~mL}$ of wastewater, suggesting that the $4 \mathrm{~S}$

264 method could be employed for future wastewater surveillance of DNA-based pathogens and NA-

265 sequencing based wastewater surveys (Supplementary Figure S3C). 
medRxiv preprint doi: https://doi.org/10.1101/2020.12.01.20242131; this version posted December 3, 2020. The copyright holder for this preprint (which was not certified by peer review) is the author/funder, who has granted medRxiv a license to display the preprint in perpetuity.

It is made available under a CC-BY-NC-ND 4.0 International license .

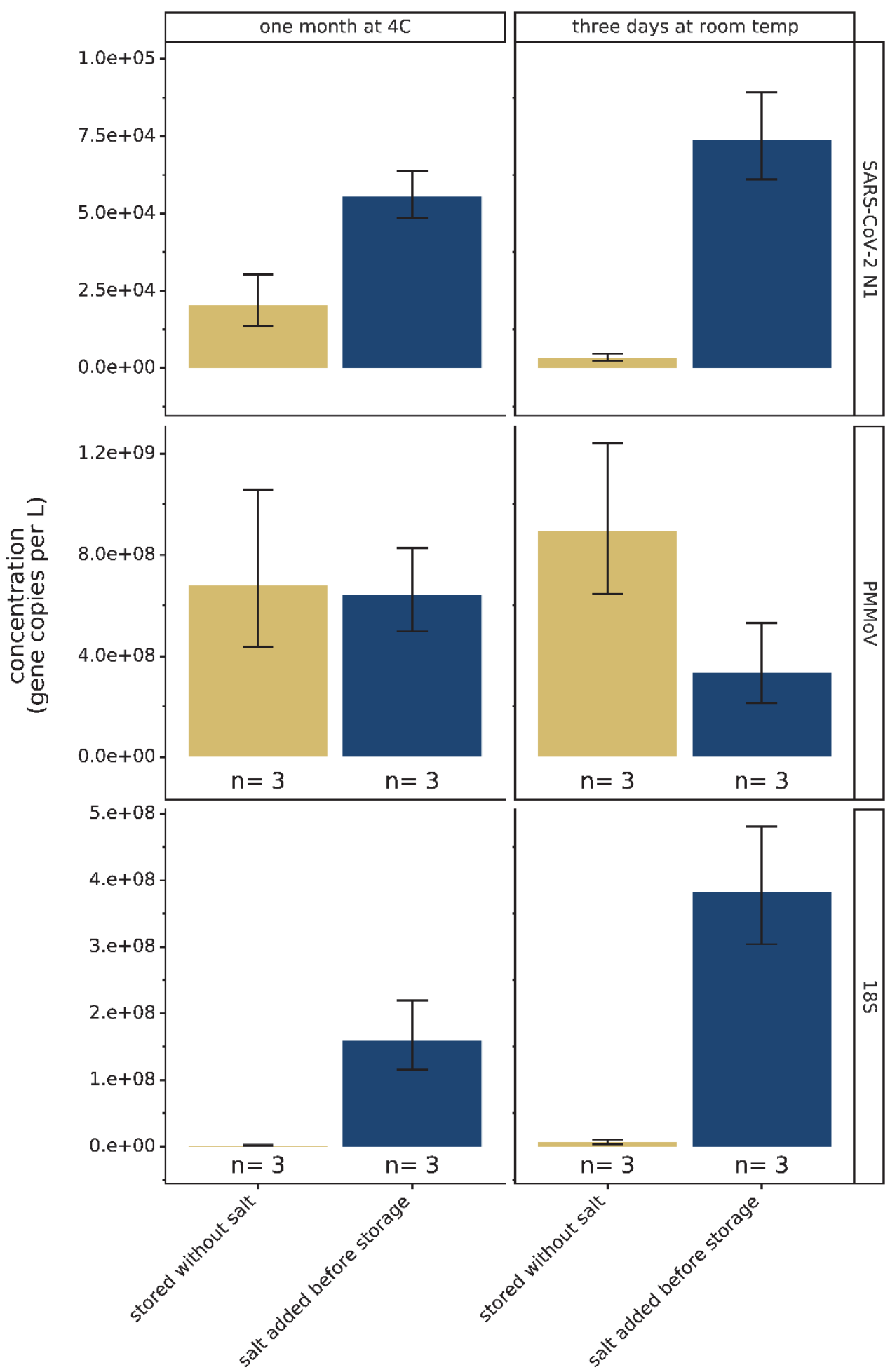

Figure 3. Effect of lysis salt addition prior to wastewater storage on SARS-CoV-2 N1, PMMoV and 18S rRNA assay signal. "n" represents the number of storage and extraction replicates per condition. Bars are plotted at the geometric mean of biological triplicates and error bars represent the variation associated with biological triplicates as quantified by the geometric standard deviation of the biological triplicates. 
Wastewater samples contain many contaminants that have previously been reported to

268 inhibit RT-qPCR reactions. ${ }^{25}$ Therefore, we sought to assess whether the 4S method could

269 generate purified RNA free of RT-qPCR contaminants by employing the "spike and dilute"

270 method to assess PCR inhibition. ${ }^{26}$ Here, we spiked purified wastewater RNA with synthetic

271 RNA standard and sequentially diluted the sample and observed whether SARS-CoV-2 N1,

$272 \mathrm{PMMoV}$, and bCoV detection followed corresponding sample dilutions, indicating an absence of

273 inhibition. We assessed the impact of a range (1-50 mL) of wash buffer volumes during RNA

274 extraction on PCR inhibition and SARS-CoV-2 N1, PMMoV, and bCoV assay signal to identify

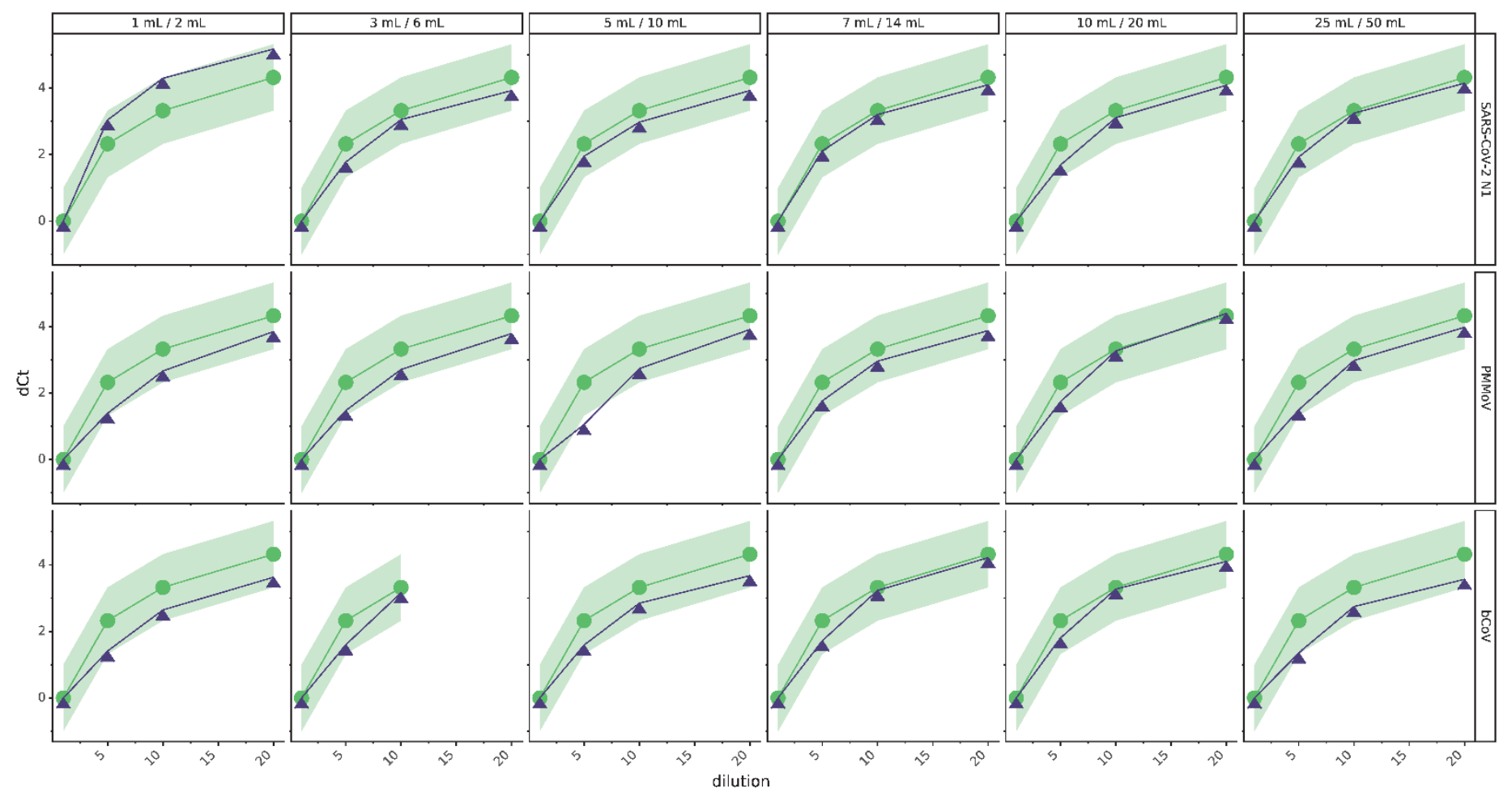

Figure 4. Assessment of RT-qPCR assay inhibition of the SARS-CoV-2 N1, PMMoV and bCoV assays via the "spike and dilute" method for different volumes of 4S-Wash buffer \#1 and 4S-Wash buffer \#2 (volumes reported at top of each panel). Sample dilutions shown are 1x, 5x, 10x, and 20x. Green line with circular points represents theoretically expected increase in $\mathrm{Ct}$ due to sample dilution, blue line with triangular points indicates actual increase in $\mathrm{Ct}$ with sample dilution. The green band indicates $+/-1 \mathrm{Ct}$ tolerance range around the expected $\mathrm{Ct}$ values, due to variability. An increase in measured $\mathrm{Ct}$ that is lower than the expected increase was interpreted as inhibition. RNA sample dilution factor is indicated on $\mathrm{x}-$ axis. 
275 the optimal wash buffer volume for RNA purity and recovery. There was no evidence of

276 inhibition for the SARS-CoV-2 N1 assay using the 4S procedure with any wash buffer volume,

277 and slight inhibition of the PMMoV assay when using $5 \mathrm{~mL}$ of 4S-Wash buffer \#1 (4S-WB1)

278 and $10 \mathrm{~mL}$ of 4S-Wash buffer \#2 (4S-WB2) (Figure 4). To limit ethanol waste generation, we

279 therefore recommend using at least $7 \mathrm{~mL}$ of $4 \mathrm{~S}-\mathrm{WB} 1$ and $14 \mathrm{~mL}$ of $4 \mathrm{~S}-\mathrm{WB} 2$ to yield inhibitor-

280 free RNA.

Next, we assessed potential assay signal loss due to excess washing of the silica columns.

282 Here, we observed highest SARS-CoV-2, PMMoV and bCoV assay signal using $3 \mathrm{~mL}$ of 4S-

283 WB1 and $6 \mathrm{~mL}$ of Wash 4S-WB2, with minimal losses in signal up until $25 \mathrm{~mL}$ of 4S-WB1 and

$28450 \mathrm{~mL}$ of 4S-WB2 (Supplementary Figure S4). Using too little wash buffer may not sufficiently

285 wash away lysis salts and contaminants from the silica matrix, reducing RNA recovery and

286 increasing inhibition, whereas too much wash buffer may partially elute bound RNA, decreasing

287 RNA yield. Therefore, we recommend using 7-10 mL of 4S-WB1 and 14-20 mL of 4S-WB2 to

288 extract PCR inhibitor-free RNA while maximizing target RNA recovery.

The results presented here are representative of only three wastewater sources which may

290 differ in composition from wastewater collected at other times and from other locations.

291 Different wastewaters may contain different types and quantities of PCR inhibitors, so we

292 recommend assessing PCR inhibition in all sample types, and if necessary, adjusting the wash

293 buffer volumes to effectively remove inhibitors from the purified RNA. Different wastewater

294 samples may also contain varying biological and chemical species influencing RNA stability,

295 potentially impacting the RNA preservation documented here by the 4S method. Furthermore,

296 the 4S method may be less effective in capturing the nucleic acids from wastewater viruses or

297 other microorganisms resistant to the sodium chloride and heat-based lysis evaluated here. 
Overall, we demonstrate that the 4S method enabled efficient extraction of SARS-CoV-2,

299 PMMoV, bCoV, and human 18S rRNA. Combined with RT-qPCR, the 4S method allowed

300 monitoring of relative COVID-19 infection prevalence with high sensitivity. These results are

301 consistent with those of a recent inter-laboratory comparison of 36 different wastewater SARS-

302 CoV-2 RNA detection methods. In this comparison, the concentration of SARS-CoV-2

303 measured with the 4S method, identified as "1S.2H", was one of the highest reported (direct

304 measurement, without correcting for recovery efficiency) and the recovery efficiency of a

305 spiked-in OC43 virus efficiency control was the highest reported, among all methods. ${ }^{27}$ The $4 \mathrm{~S}$

306 method also preserved RNA in wastewater, was compatible with heat pasteurization, and yielded

307 purified RNA free of PCR inhibitors. Given the high efficiency, low cost, and same-day

308 assessment of wastewater SARS-CoV-2 and fecal concentration controls, the 4S method presents

309 an affordable and accessible method for implementing wastewater-based epidemiology for

310 SARS-CoV-2. The method also appears promising for the application of WBE for other RNA-

311 and DNA-based pathogens and facilitating research on the wastewater microbial community

312 more broadly. 


\section{$\underline{\text { Acknowledgements }}$}

320 We thank all members of the Nelson laboratory and UC Berkeley COVID-WEB pop-up lab for

321 their contributions, reading, insights and helpful discussions of this paper. We also thank all

322 members of the Tjian and Darzacq lab for their support designing and carrying out molecular

323 assays and experiments. We thank the East Bay Municipal Utility District for sample collection.

\section{$\underline{\text { Funding sources }}$}

326 O.N.W is supported by the NIH training program grant T32GM007232. We gratefully

327 acknowledge funding from the Howard Hughes Medical Institute (grant CC34430 to R.T.) and

328 from rapid response grants from the Center for Information Technology Research in the Interest

329 of Society (CITRIS) and the Innovative Genomics Institute (IGI) at UC Berkeley to K.L.N.

$330 \quad 000$

\section{$331 \quad \underline{\text { References }}$}

332 1. Bivins, A. et al. Wastewater-Based Epidemiology: Global Collaborative to Maximize

333 Contributions in the Fight Against COVID-19. Environ. Sci. Technol. 54, 7754-7757 (2020).

334 2. Zhou, N. A. et al. Feasibility of the Bag-Mediated Filtration System for Environmental

335 Surveillance of Poliovirus in Kenya. Food Environ Virol 12, 35-47 (2020).

336 3. Kazama, S. et al. Environmental Surveillance of Norovirus Genogroups I and II for

337 Sensitive Detection of Epidemic Variants. Appl. Environ. Microbiol. 83, (2017).

338 4. Kaliner, E. et al. The Israeli public health response to wild poliovirus importation. The

339 Lancet Infectious Diseases 15, 1236-1242 (2015).

340 5. Hata, A. \& Honda, R. Potential Sensitivity of Wastewater Monitoring for SARS-CoV-2:

341 Comparison with Norovirus Cases. Environ. Sci. Technol. (2020) doi:10.1021/acs.est.0c02271. 
342 6. Li, R. et al. Substantial undocumented infection facilitates the rapid dissemination of

343 novel coronavirus (SARS-CoV-2). Science 368, 489-493 (2020).

3447 Wu, Y. et al. Prolonged presence of SARS-CoV-2 viral RNA in faecal samples. The

345 Lancet Gastroenterology \& Hepatology 5, 434-435 (2020).

346 8. Holshue, M. L. et al. First Case of 2019 Novel Coronavirus in the United States. New

347 England Journal of Medicine 382, 929-936 (2020).

3489 9. Peccia, J. et al. Measurement of SARS-CoV-2 RNA in wastewater tracks community

349 infection dynamics. Nature Biotechnology 38, 1164-1167 (2020).

350 10. Ahmed, W. et al. First confirmed detection of SARS-CoV-2 in untreated wastewater in

351 Australia: A proof of concept for the wastewater surveillance of COVID-19 in the community.

352 Science of The Total Environment 138764 (2020) doi:10.1016/j.scitotenv.2020.138764.

353 11. Medema, G., Heijnen, L., Elsinga, G., Italiaander, R. \& Brouwer, A. Presence of SARS-

354 Coronavirus-2 RNA in Sewage and Correlation with Reported COVID-19 Prevalence in the

355 Early Stage of the Epidemic in The Netherlands. Environ. Sci. Technol. Lett. 7, 511-516 (2020).

356 12. Crits-Christoph, A. et al. Genome sequencing of sewage detects regionally prevalent

357 SARS-CoV-2 variants. medRxiv 2020.09.13.20193805 (2020)

358 doi:10.1101/2020.09.13.20193805.

359 13. Kitajima, M. et al. SARS-CoV-2 in wastewater: State of the knowledge and research

360 needs. Science of The Total Environment 739, 139076 (2020).

361 14. La Rosa, G., Bonadonna, L., Lucentini, L., Kenmoe, S. \& Suffredini, E. Coronavirus in

362 water environments: Occurrence, persistence and concentration methods - A scoping review.

363 Water Research 179, 115899 (2020). 
364 15. Lu, D., Huang, Z., Luo, J., Zhang, X. \& Sha, S. Primary concentration - The critical step

365 in implementing the wastewater based epidemiology for the COVID-19 pandemic: A mini-

366 review. Science of The Total Environment 747, 141245 (2020).

367 16. Shortage of RNA extraction kits hampers efforts to ramp up COVID-19 coronavirus

368 testing. Chemical \& Engineering News https://cen.acs.org/analytical-

369 chemistry/diagnostics/Shortage-RNA-extraction-kits-hampers/98/web/2020/03.

370 17. CDC. Coronavirus Disease 2019 (COVID-19). Centers for Disease Control and

371 Prevention https:/www.cdc.gov/coronavirus/2019-ncov/cases-updates/wastewater-

372 surveillance/testing-methods.html (2020).

373 18. Kitajima, M., Sassi, H. P. \& Torrey, J. R. Pepper mild mottle virus as a water quality

374 indicator. npj Clean Water 1, 1-9 (2018).

375 19. Wang, X.-W. et al. Concentration and detection of SARS coronavirus in sewage from

376 Xiao Tang Shan Hospital and the 309th Hospital. Journal of Virological Methods 128, 156-161

377 (2005)

378 20. C. Wetter, M. Conti, D. Altschuh, R. Tabillon, and M.H.V. van Regenmortel. Pepper

379 Mild Mottle Virus, a Tobamovirus Infecting Pepper Cultivars in Sicily. Phytopathology (1983).

380 21. Bivins, A. et al. Persistence of SARS-CoV-2 in Water and Wastewater. Environ. Sci.

381 Technol. Lett. acs.estlett.0c00730 (2020) doi:10.1021/acs.estlett.0c00730.

382 22. Ahmed, W. et al. Decay of SARS-CoV-2 and surrogate murine hepatitis virus RNA in

383 untreated wastewater to inform application in wastewater-based epidemiology. Environmental

384 Research 191, 110092 (2020). 
23. Phillips, S. J., Dalgarn, D. S. \& Young, S. K. Recombinant DNA in Wastewater: pBR322 Degradation Kinetics. Research Journal of the Water Pollution Control Federation 61, 15881595 (1989).

24. Michael-Kordatou, I., Karaolia, P. \& Fatta-Kassinos, D. Sewage analysis as a tool for the COVID-19 pandemic response and management: the urgent need for optimised protocols for

390 SARS-CoV-2 detection and quantification. J Environ Chem Eng 8, 104306 (2020).

391 25. Schrader, C., Schielke, A., Ellerbroek, L. \& Johne, R. PCR inhibitors - occurrence, 392 properties and removal. Journal of Applied Microbiology 113, 1014-1026 (2012).

393 26. Cao, Y., Griffith, J. F., Dorevitch, S. \& Weisberg, S. B. Effectiveness of qPCR

394 permutations, internal controls and dilution as means for minimizing the impact of inhibition

395 while measuring Enterococcus in environmental waters. Journal of Applied Microbiology 113, $396 \quad 66-75(2012)$.

397 27. Pecson, B. M. et al. Reproducibility and sensitivity of 36 methods to quantify the SARS398 CoV-2 genetic signal in raw wastewater: findings from an interlaboratory methods evaluation in 399 the U.S. medRxiv 2020.11.02.20221622 (2020) doi:10.1101/2020.11.02.20221622.

400 28. Whitney, O. Direct wastewater RNA capture and purification via the \&\#34;Sewage, Salt, 401 Silica and SARS-CoV-2 (4S)\&\#34; method. (2020) doi:10.17504/protocols.io.biwekfbe.

402 29. Whitney, O. Direct wastewater RNA extraction via the \&\#34;Milk of Silica (MoS)\&\#34; 403 method - A companion method to \&\#34;Sewage, Salt, Silica and SARS-CoV-2 (4S)\&\#34;

404 (2020) doi:10.17504/protocols.io.biwfkfbn.

405 30. Graham, T. G. W. et al. Inexpensive, versatile and open-source methods for SARS-CoV-2 406 detection. http://medrxiv.org/lookup/doi/10.1101/2020.09.16.20193466 (2020)

407 doi:10.1101/2020.09.16.20193466. 
408 31. Haramoto, E. et al. Occurrence of Pepper Mild Mottle Virus in Drinking Water Sources

409 in Japan. Appl. Environ. Microbiol. 79, 7413-7418 (2013).

410 32. Decaro, N. et al. Detection of bovine coronavirus using a TaqMan-based real-time RT-

411 PCR assay. Journal of Virological Methods 151, 167-171 (2008).

412 33. Bustin, S. A. et al. The MIQE Guidelines: Minimum Information for Publication of

413 Quantitative Real-Time PCR Experiments. Clin Chem 55, 611-622 (2009).

414 34. Wu, F. et al. SARS-CoV-2 Titers in Wastewater Are Higher than Expected from

415 Clinically Confirmed Cases. 5, 9 (2020).

416 35. Haramoto, E., Malla, B., Thakali, O. \& Kitajima, M. First environmental surveillance for

417 the presence of SARS-CoV-2 RNA in wastewater and river water in Japan. Sci Total Environ

418737,140405 (2020).

419 36. Alameda County COVID-19 Daily Cumulative Cases by City, Place, and Zip Code.

420 https://data.acgov.org/datasets/5d6bf4760af64db48b6d053e7569a47b_3?page=10. 